Vol. 16, Núm. 2

Abr.-Jun. 2020. pp 54-61 doi: $10.35366 / 94533$

https://dx.doi.org/10.35366/94533

\title{
Coronavirus, la epidemia que cambió el mundo
}

\author{
Coronavirus, the epidemic that changed the world
}

Leonardo López Almejo, ${ }^{*}$ Luis Gerardo Padilla Rojas, ${ }^{\ddagger}$
Darío Esaú Garín Zertuche, ${ }^{\S}$ Michael Dittmar Johnson"

\author{
* Vicepresidente FEMECOT, Coordinador del Comité Científico FEMECOT. Cirujano Ortopedista, \\ Cirujano de Mano. Profesor titular del Diplomado en Cirugía de Plexo Braquial \\ y Nervio periférico. Universidad Autónoma de Coahuila. \\ ₹ Tesorero FEMECOT, Coordinador del módulo de Trauma del Comité Científico FEMECOT. Cirujano articular. \\ \& Coordinador del módulo de Cadera del Comité Científico FEMECOT. Cirujano articular. \\ " Coordinador del módulo de Columna del Comité Científico FEMECOT. Cirujano de columna.
}

Correspondencia: Leonardo López Almejo. Av. Universidad No. 103, Interior 219,

Col. Villas de la Universidad, 20020, Aguascalientes, Ags. México.

Tel: +52 4494139901. Correo electrónico: lyon77777@gmail.com.

\section{RESUMEN}

En diciembre de 2019 se produjo una nueva enfermedad en China que ha presentado una diseminación global, afectando actualmente a más de 4 millones y produciendo la muerte a más de 300 mil personas, con una tasa de mortalidad que va por arriba de $13 \%$ en varios países en Europa. La mayoría de los pacientes tienen síntomas leves y buen pronóstico. Las manifestaciones más comunes son fiebre, tos seca, disnea, mialgia, cefalea y fatiga. Otros hallazgos son disminución de linfocitos en la sangre, alto índice de ferritina y evidencia radiográfica de neumonía, con infiltrado atípico por daño alveolar difuso, inflamación intersticial que se cree sea un tromboembolismo. Hay afectación a varios órganos, y en muchos casos una coagulación intravascular diseminada (CID), con afección del endotelio vascular. El estrés asociado con la fractura y el tratamiento quirúrgico pueden generar una respuesta de estrés oxidativo. Aumentando la expresión de factores inflamatorios y disminuyendo la inmunidad del paciente,

\section{ABSTRACT}

In December 2019 a new disease occurred in China that has presented a global spread. Currently affecting more than 4 million and causing the death of more than 300 thousand people. With a mortality rate that goes above $13 \%$ in several countries in Europe. Most patients have mild symptoms and a good prognosis. The most common manifestations are fever, dry cough, dyspnea, myalgia, headache, fatigue. Other findings are a decrease in lymphocytes in the blood, a high ferritin index and radiographic evidence of pneumonia, with an atypical infiltrate due to diffuse alveolar damage, believed to be an interstitial inflammation, thromboembolism. There is involvement of various organs, and in many cases disseminated intravascular coagulation (DIC), with involvement of the vascular endothelium. The stress associated with fracture and surgical treatment can generate an oxidative stress response. Increasing the expression of inflammatory factors and decreasing the 
lo cual incrementa el riesgo de muerte por COVID-19. El objetivo de este documento es ser una guía para el Ortopedista para cuidar y proteger al equipo quirúrgico y al paciente, conociendo los riesgos del tratamiento quirúrgico, el buen uso y manejo del equipo de colocación y el material de osteosíntesis, y los procedimientos quirúrgicos que se pueden realizar durante esta pandemia, con el objetivo de disminuir riesgos de contagio y muerte en pacientes y en el personal de la salud.

Palabras clave: COVID-19, coronavirus, cirugía ortopédica en pandemia, daño por COVID-19, fractura e infección por coronavirus. patient's immunity, increasing the risk of death from COVID-19. The objective of this document is to be a guide for the Orthopedist to care for and protect the surgical team and the patient, knowing the risks of surgical treatment, good use and management of the placement equipment and osteosynthesis material. And the surgical procedures that can be performed during this pandemic, with the aim of reducing risks of contagion and death in patients and in health workers.

Keywords: COVID-19, coronavirus, pandemic orthopedic surgery, COVID-19 damage, coronavirus fracture and infection.

El 08 de diciembre del 2019 se produjo un brote de una nueva enfermedad, mostrando casos de neumonía de etiología desconocida en Wuhan, Hubei, China. ${ }^{1-3}$

El 07 de enero del 2020, el Centro Chino para el Control y la Prevención de Enfermedades (CDC) identificó un nuevo tipo de virus de la familia Coronaviridae. Fue denominado 2019-nCoV por la OMS, También nombrado Coronavirus SARS 2 (SARS-CoV-2) (COVID-19) 4,5 (Figura 1).

El 10 de enero del 2020, ocurrió la primera muerte en China. El 13 de enero se identificó el primer caso en Tailandia, en Japón y Corea el 15 y 20 de enero, respectivamente. ${ }^{6,7}$ Después se presentó una diseminación global.

El 11 de marzo del 2020 la OMS declara «Pandemia» a la enfermedad por COVID-19. ${ }^{8}$

El Gobierno de México, a través de la Secretaría de Salud, reportó 51,633 casos confirmados y 5,332 muertes el 18 de mayo del 2020. La Ciudad de México, Estado de México, Baja California Tabasco, Sinaloa y Veracruz son los Estados más afectados. La enfermedad predomina en hombres $(57.72 \%)$ y las principales comorbilidades son hipertensión $(21.6 \%)$, obesidad $(20.74 \%)$ y diabetes $(18.32 \%)^{9}$ (Figura 2).

Hasta el 18 de mayo del 2020 se han documentado 4,801,282 casos confirmados en el mundo y 318,465 muertes, afectando principalmente a Estados Unidos, Rusia, Brasil, Reino Unido, España, Italia, Francia, Alemania y Turquía. Se consideró que existía una transmisión comunitaria de China continental a los demás países, como Singapur, Hong-Kong, Japón, Corea del Sur, Tailandia, Italia e Irán (Figura 3).

Inicialmente, la tasa de mortalidad fue de $3 \%$. Actualmente en países como Bélgica, Francia, Italia y Reino Unido ha rebasado 13\%. En México representa $10 \%$ al día 09 de mayo del 202010,11 (Figura 4).

EI SARS-CoV-2 es un Coronaviridae de la subfamilia Ortho-Coronavirinae. Se han descrito siete tipos que afectan a seres humanos (HCoV-229E, HCoVNL63, HCoV-OC43, HCoV-HKU1, SARS-CoV, MERS-CoV y SARS-CoV-2). ${ }^{10,12,13}$ 
A)

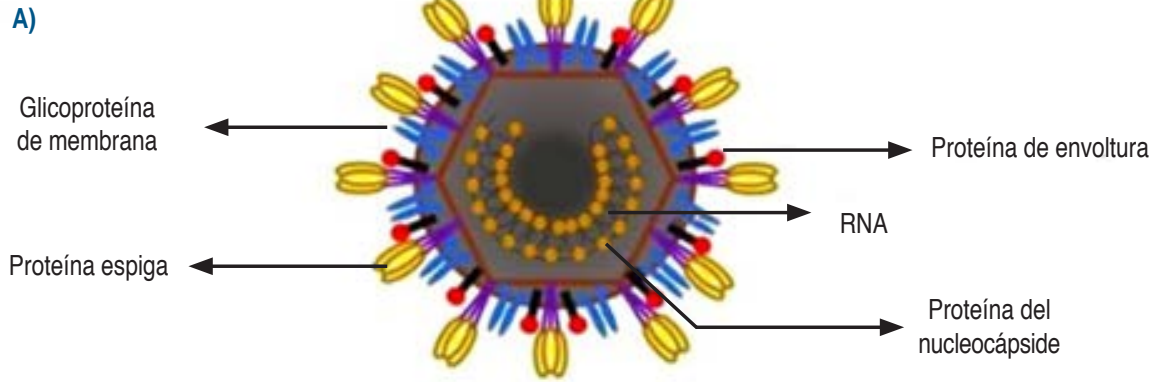

B)

SARS-COV
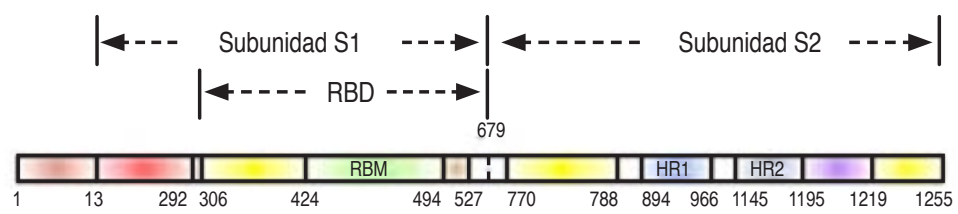

687

SARS-CoV-2

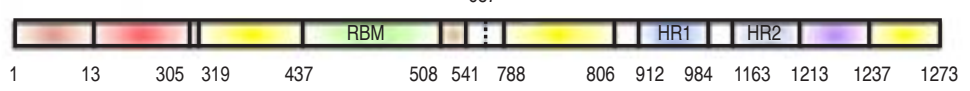

Figura 1: Representación esquemática del coronavirus, su membrana y proteínas. A) Estructura del coronavirus. Las proteínas de la superficie viral (glicoproteínas de membrana) están incrustadas en una envoltura de bicapa lipídica. B) Comparación de las proteínas de SARS-CoV y SARS-CoV-2. Modificado de: Zhou G, Zhao Q. Perspectives on therapeutic neutralizing antibodies against the Novel Coronavirus SARS-CoV2. Int J Biol Sci. 2020; 16 (10): 1718-1723. doi:10.7150/ijbs.45123.

\section{COVID-19 México}

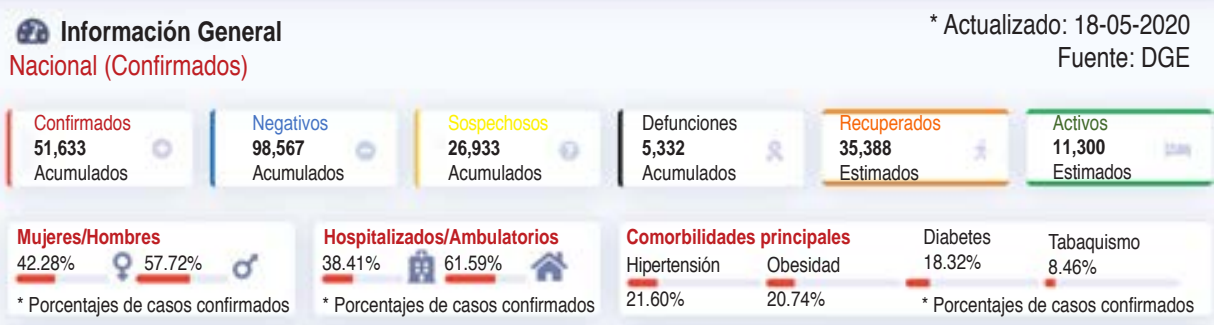

Figura 2: Esquema que representa la evolución de la pandemia en México del 28 de febrero al 18 de mayo del 2020. Tomado de: Comunicado Técnico Diario SSA. Gobierno de México, 2020.

Con base en los datos de inicio de los síntomas, la duración de la estancia e historial de viajes en los pacientes de Wuhan, que fueron confirmados con infección por coronavirus, se obtuvo un rango de valores posibles para el periodo de incubación, ajustando tres formas paramétricas, por lo que se considera la posibilidad de periodos de incubación de 6.2 hasta 15.2 días en esta etapa de la epidemia ${ }^{14}$ (Figura 5). 


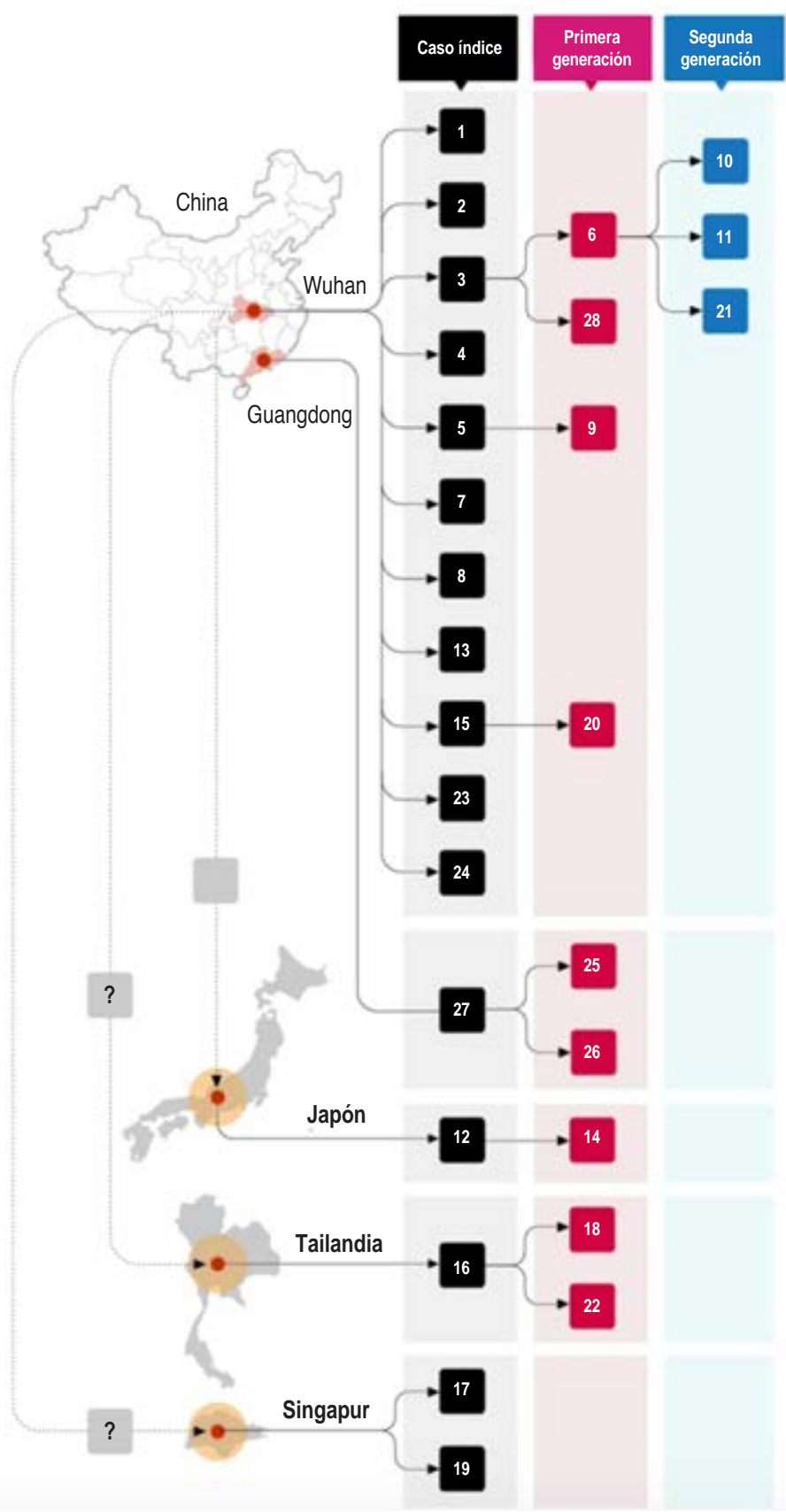

Figura 3: Ejemplo de una distribución comunitaria de la transmisión viral de los primeros casos en Corea según la cadena de transmisión.

Modificado de: Moran K. Epidemiologic characteristics of early cases with 2019 novel coronavirus (2019-nCoV) disease in Korea. Special article. Epidemiol Health. 2020; 42: e2020007. Available in: https://doi.org/10.4178/epih.e2020007. 


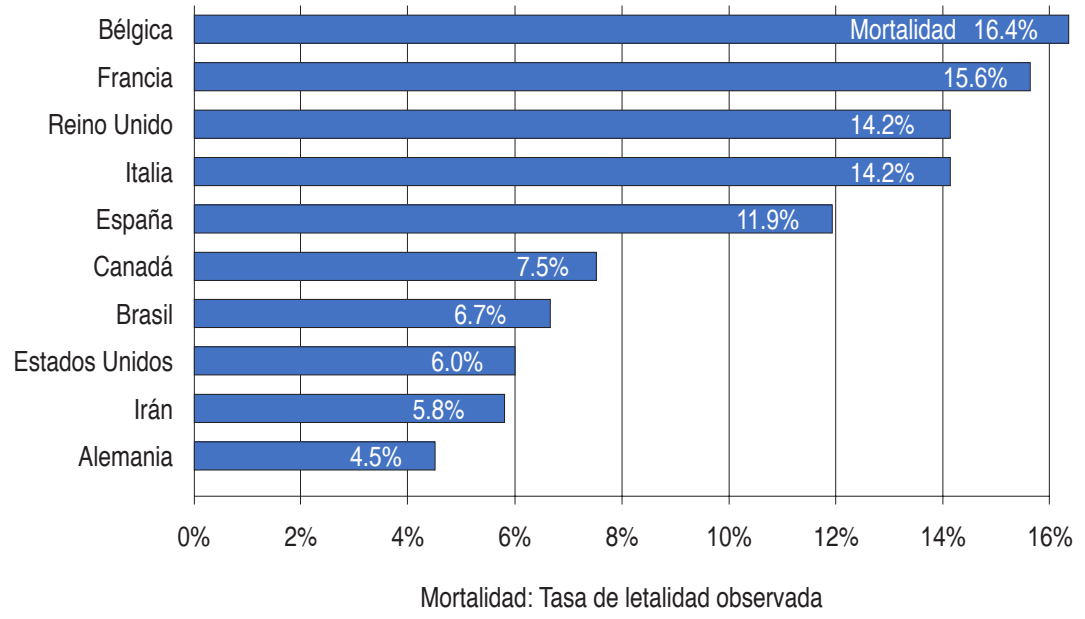

Figura 4: Relación de la tasa de mortalidad en diferentes países. Modificado de: https://coronavirus.jhu.edu/data/mortality.

La mayoría de los pacientes tienen síntomas leves y buen pronóstico. Las manifestaciones más comunes son fiebre, tos seca, disnea, mialgia, cefalea, fatiga. Otros hallazgos son disminución de linfocitos en la sangre, alto índice de ferritina y evidencia radiográfica de neumonía, con infiltrado atípico por daño alveolar difuso, inflamación intersticial que se cree sea un tromboembolismo. Hay afectación a varios órganos, y en muchos casos una coagulación intravascular diseminada (CID), con afección del endotelio vascular. ${ }^{15}$

Las complicaciones son: síndrome de dificultad respiratoria aguda, arritmia, choque, lesión cardiaca aguda, infección secundaria e insuficiencia renal aguda, neumonía severa, edema pulmonar, insuficiencia orgánica múltiple. Comportándose como una enfermedad sistémica, que conlleva a la muerte..$^{5,16}$

No todas las personas que se han expuesto al virus han desarrollado la enfermedad. ${ }^{17}$ Los pacientes con fracturas graves requieren tratamiento quirúrgico de urgencia. Los adultos mayores con fractura de cadera tienen comorbilidades como: hipertensión, diabetes o enfermedades cardiacas, haciendo a esta población más susceptible a COVID-19. ${ }^{18}$ El estrés asociado con la fractura y el tratamiento quirúrgico pueden generar una respuesta de estrés oxidativo, lo que aumenta la expresión de factores inflamatorios y disminuye la inmunidad del paciente, incrementando el riesgo de neumonía por COVID-19. El recuento medio de neutrófilos y el dímero $D$, las citoquinas y las quimiocinas proinflamatorias (factor de necrosis tumoral (TNF) $\alpha$, interleucina $1 \beta$ (IL-1 $\beta$ ), IL-6, factor estimulante de colonias de granulocitos, proteína 10 inducida por interferón gamma, proteína 1 de monocitos y proteínas inflamatorias de macrófagos 1 - $\alpha$ ), se elevaron significativamente en pacientes con COVID-19. ${ }^{15,18-21}$

La respuesta humoral mediada por anticuerpos es crucial para prevenir infecciones virales. Un subconjunto de estos anticuerpos, que reducen la infectivi- 
dad viral al unirse a los epítopos de la superficie de las partículas virales y, por lo tanto, bloquean la entrada del virus a una célula infectada, se definen como anticuerpos neutralizantes (NAbs). ${ }^{22}$ Los NAbs obtienen sus actividades de protección en tres pasos principales: 1. Evitando la unión del virión a sus receptores en las células objetivo. 2. Causando la agregación de partículas virales. 3. Lisando al virus a través de la región constante $(\mathrm{C})$ de la opsonización mediada por anticuerpos o la activación del complemento.

Por lo tanto, sigue siendo importante comprender la inmunopatogénesis del SARS-CoV-2 y abordar los beneficios, desafíos y consideraciones de los anticuerpos neutralizantes (NAbs). Por consiguiente, es significativo conocer la respuesta humoral y celular del SARS-CoV-2 cuando se desarrolla la inmunoterapia antiviral ${ }^{23,24}$ (Figura 6). Más aún en pacientes que han sufrido una fractura y pueden mostrar alteración de estos sistemas por el proceso inflamatorio constante que viven.

Las manos tienen potencial de trasmisión del COVID-19, está perfectamente establecido, por lo que todo paciente traumatizado, en choque o inconsciente, se le debe realizar un protocolo de aseo en sus manos, boca y nariz, y realizarles la prueba de isopo nasal y retrofaringeo. ${ }^{18,25}$

El objetivo esencial de este documento es proporcionar al lector Ortopedista o profesional de cualquier área de la salud un recurso que le permita de una manera sencilla entender la severidad de la pandemia por SARS-CoV-2 y realizar un análisis sobre cómo cuidar y proteger al equipo quirúrgico y al paciente, entendiendo la fisiopatología de las lesiones y los riesgos que conlleva al realizar un tratamiento quirúrgico en esta etapa, así como el buen uso y manejo del instrumental especializado y el material de osteosíntesis.

Además de entender el proceder del cirujano en el quirófano y cuáles son los procedimientos quirúrgicos que se pueden realizar durante esta pandemia en sus diferentes fases, con el objetivo de disminuir riesgos de contagio y las complicaciones que esto conlleva y, como siempre ha sido primordial para el cirujano ortopedista, preservar la vida y mejorar la función de un paciente.

Finalmente, hay que recordar que leer es un signo de salud científica, y los médicos que investigan tienen un mejor desempeño con sus pacientes y con su comunidad.

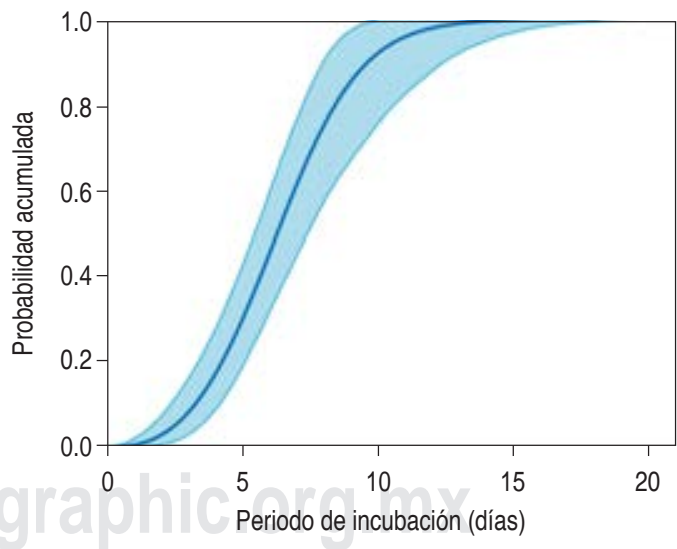

Figura 5: Tabla que representa el periodo de incubación del SARS-COV-2.

Modificado de: Backer JA, Klinkenberg D, Wallinga J. Incubation period of 2019 novel coronavirus (2019-nCoV) infections among travellers from Wuhan, China. Euro Surveill. 2020;25(5):2000062. doi: 10.2807/1560-7917. 

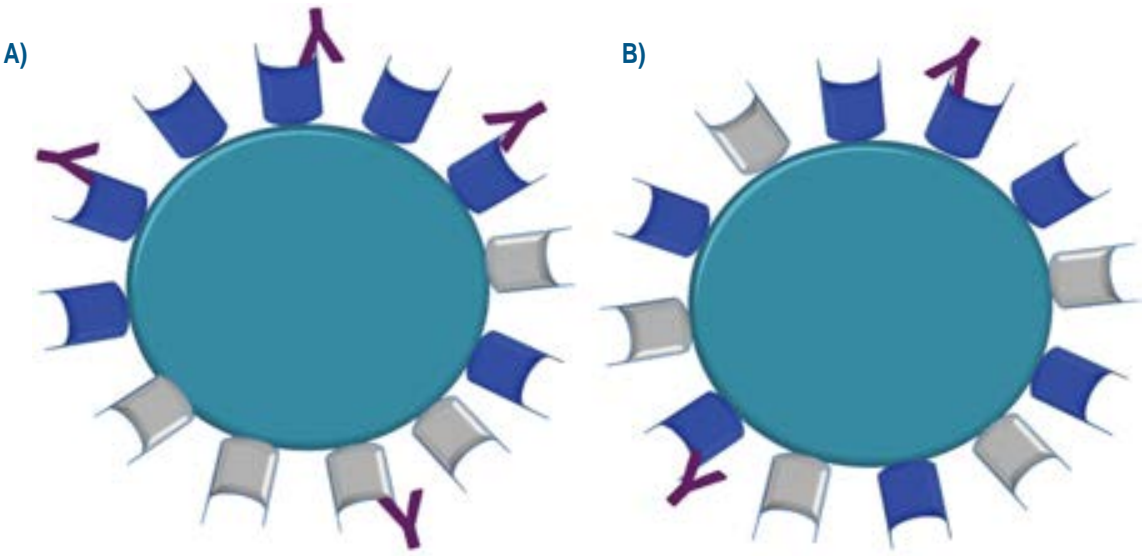

Figura 6: Neutralizando las ocupaciones sobre poblaciones heterogéneas de virus envueltos. Se muestran dos viriones envueltos. Cada uno tiene doce picos de glucoproteína mostrados esquemáticamente. Los picos funcionales se muestran en azul, cariados o no funcionales en gris. Ambos viriones tienen siete picos funcionales y cinco no funcionales, pero con diferentes distribuciones sobre las dos superficies del virión. Si un virión requiere un cierto número de picos en contigüidad para formar un complejo de entrada y los picos no pueden moverse libremente sobre

la superficie del virión, las dos distribuciones diferentes conferirán sensibilidades de neutralización diferentes.

El virión a la izquierda se neutraliza: tres moléculas de NAb desactivan la constelación de espigas activas y una se une de forma redundante a una espiga inactiva. El virión a la derecha también está

neutralizado, pero sólo por dos moléculas de NAb, una inactivando un grupo de tres espigas (tres postulantes adyacentes se postulan aquí como el mínimo para la entrada) y una que se une de forma redundante a una espiga que es funcional pero inerte a través de falta de vecinos activos.

Tomado de: Klasse PJ. Neutralization of virus infectivity by antibodies: old problems in new perspectives. Adv Biol. 2014; 2014: 157895.

\section{BIBLIOGRAFÍA}

1. Wang D, Hu B, Hu C, Zhu F, Liu X, Zhang J, et al. Clinical characteristics of 138 hospitalized patients with 2019 novel coronavirus-infected pneumonia in Wuhan, China. JAMA. 2020; 323 (11): 1061-1069.

2. Zhou P, Yang XL, Wang XG, Hu B, Zhang L, Zhang W, et al. A pneumonia outbreak associated with a new coronavirus of probable bat origin. Nature. 2020; 579 (7798): 270-273.

3. Wu JT, Leung K, Leung GM. Nowcasting and forecasting the potential domestic and international spread of the 2019-nCoV outbreak originating in Wuhan, China: a modelling study. Lancet. 2020; 395: 689-697.

4. Zhu N, Zhang D, Wang W, Li X, Yang B, Song J, et al. A novel coronavirus from patients with pneumonia in China. N Engl J Med. 2020; 382 (8): 727-733.

5. Nanshan C, Min Z, Xuan D, Jieming Q, Fengyun G, Yang H, et al. Epidemiological and clinical characteristics of 99 cases of 2019 novel coronavirus pneumonia in Wuhan, China: a descriptive study. Lancet. 2020; 395: 507-513.

6. Yoo JH, Hong ST. The outbreak cases with the novel coronavirus suggest upgraded quarantine and isolation in Korea. J Korean Med Sci. 2020; 35 (5): e62.

7. Moran K. Epidemiologic characteristics of early cases with 2019 novel coronavirus (2019-nCoV) disease in Korea. Special article. Epidemiol Health. 2020; 42: e2020007.

8. Chang-Liang Z, Wang W, Murphy D, Hoi-Po-Hui J. Novel coronavirus and orthopedic surgery. Early experiences from Singapore. J Bone Joint Surg Am. 2020; 102: 745-749.

9. López-Gatell RH. Comunicado Técnico Diario. Unidad de inteligencia Epidemiológica y Sanitaria (Internet). Subsecretaría de Prevención y Promoción de la Salud. Secretaría de Salud. Gobierno de México 2020. (Citado el 09 de mayo 2020). Disponible en https://www.gob.mx/salud.

10. Montero FA, Maseda E, Adalia BR, Aguilar G, González de Castro R, Gómez-Herreras Jl, et al. Recomendaciones prácticas para el manejo perioperatorio del paciente con sospecha o infección grave por coronavirus SARS-CoV2. Rev Esp Anestesiol Reanim. 2020; 67 (5): 253-260. 
11. University of Medicine Johns Hopkins. Coronavirus Resource Center. COVID-19 Case Tracker (Internet). Baltimore, Maryland. Estados Unidos. https://www.jhu.edu. 2020. (Actualizado 09 de mayo 2020). Available in: https://coronavirus.jhu.edu.

12. Holshue ML, DeBolt C, Lindquist S, Lofy KH, Wiesman J, Bruce H, et al. Washington State 2019-nCoV case investigation team. First case of 2019 novel coronavirus in the United States. N Engl J Med. 2020; 382 (10): 929-936.

13. Wu Z, McGoogan JM. Characteristics of and important lessons from the coronavirus disease 2019 (COVID-19) outbreak in China: summary of a report of 72,314 cases from the Chinese center for disease control and prevention. JAMA. 2020; 323 (13): 1239-1242.

14. Backer JA, Klinkenberg D, Wallinga J. Incubation period of 2019 novel coronavirus (2019-nCoV) infections among travellers from Wuhan, China. Euro Surveill. 2020; 25 (5): 2000062. doi: 10.2807/1560-7917.

15. Li H, Liu L, Zhang D, Xu J, Dai H, Tang N, et al. SARS-CoV-2 and viral sepsis: observations and hypotheses. Lancet. 2020; PubMed PMID: 32311318.

16. Shaoqing, L, Fang J, Wating S, Chang C, Jingli C, Wei M, et al. Clinical characteristics and outcomes of patients undergoing surgeries during the incubation period of COVID-19 infection. E Clinical Medicine (2020). Article in press.

17. Phan LT, Nguyen TV, Luong QC, Nguyen TV, Nguyen HT. Importation and human-to-human transmission of a novel coronavirus in Vietnam. N Engl J Med. 2020; 382 (9): 872-874.

18. Bobin M, Lang C, Yuan X, Hang X, Wu Z, Guohui L. Characteristics and early prognosis of COVID-19 infection in fracture patients. J Bone Joint Surg Am. 2020; 102 (9): 750-758.

19. HIP ATTACK Investigators. Accelerated surgery versus standard care in hip fracture (HIP ATTACK): an international, randomised, controlled trial. Lancet. 2020; 29: 395(10225):698-708.

20. Lizaur UA, Lopez PFA. Hip attack for hip fractures: is ultra-early surgery necessary? Lancet. $2020 ; 395$ (10225): 661-662.

21. Ravi B, Pincus D, Wasserstein D, Govindarajan A, Huang A, Austin PC, et al. Association of overlapping surgery with increased risk for complications following hip surgery: a population-based, matched cohort study. JAMA Intern Med. 2018; 178 (1): 75-83.

22. Klasse PJ. Neutralization of virus infectivity by antibodies: old problems in new perspectives. Adv Biol. 2014; 2014: 157895. doi: 10.1155/2014/157895.

23. Coughlin MM, Prabhakar BS. Neutralizing human monoclonal antibodies to severe acute respiratory syndrome coronavirus: target, mechanism of action, and therapeutic potential. Rev Med Virol. 2012; 22: 2-17.

24. Zhou G, Zhao Q. Perspectives on therapeutic neutralizing antibodies against the Novel Coronavirus SARS-CoV-2. Int J Biol Sci. 2020; 16 (10): 1718-1723. doi: 10.7150/ijbs.45123.

25. De Vitis R, Passiatore M, Perna A, Proietti L, Taccardo G. COVID-19 contagion and contamination through hands of trauma patients: what risks and what precautions? J Hosp Infect. 2020; 105(2): 354-355.

\section{Código ORCID:}

López Almejo 0000-0002-6104-2349.

Padilla Rojas 0000-0001-6658-4123. 